\title{
Quale infrastruttura per le edizioni digitali?
}

\author{
Dalla tecnologia all'etica
}

\author{
Elena Pierazzo
}

\begin{abstract}
L'articolo riflette sui modi di produzione delle edizioni scientifiche digitali, lamentandone $i$ costi eccessivi che di fatto escludono i giovani ricercatori dalla loro produzione. Utilizzando una metafora presa in prestito dal mondo della moda, le edizioni scientifiche digitali esistenti sono paragonate all'haute couture e al prêt-à-porter: l'autrice distingue infatti le edizioni specializzate, caratterizzate dai costi elevati e dall'essere costruite "su misura" (haute couture) da quelle seriali, caratterizzate da una certa uniformità e dai costi ridotti (prêt-àporter), auspicando una più generale diffusione di quest'ultimo modello. La seconda parte dell'articolo riflette sulle caratteristiche delle edizioni seriali e loro relazioni con le edizioni specializzate.
\end{abstract}

This article reflects on current ways of producing digital scholarly editions, noting that they typically involve excessive costs that effectively exclude young or independent scholars from undertaking their production. Using a metaphor borrowed from the world of fashion, existing digital scholarly editions are described as either 'haute couture' or 'prêt-à-porter': that is, either specialized editions characterized by high costs and by production 'to measure' (haute couture), or those that use existing frameworks and so are characterized by a certain uniformity and reduced costs (prêt-à-porter). The article argues for a wider diffusion of the latter model, but the second part presents a reflection on the characteristics of these editions and their productive interactions with the more specialized type of edition.

\section{$\mathrm{L}_{\mathrm{A}}$}

CREAZIONE DI EDIZIONi DIGITAli SCIENTIFICHE ALTAMENTE sofisticate e su misura ha caratterizzato la fase "pionieristica" della filologia digitale; tuttavia, nonostante l'eccellenza dei risultati scientifici prodotti da molte di quelle edizioni, ci sono diversi motivi per considerare le edizioni prodotte finora come meno che soddisfacenti da molti punti di vista. Il problema principale consiste nel fatto che ogni edizione digitale presenta il testo in modi diversi, a volte riconducibili a tipi di edizioni tradizionali come le edizioni diplomatiche o critiche, a volte seguendo modelli inno- 
vativi e senza precedenti che offrono diversi tipi di testualità e interazioni ai loro utenti spesso disorientati dalle troppe scelte disponibili; tali diversità sono presenti nonostante (e forse grazie a) una forte uniformità dei dati, visto che la maggior parte delle edizioni utilizza il formato sviluppato dalla Text Encoding Initiative (TEI Consortium 2018). Inoltre, da un punto di vista tecnico le edizioni sono offerte da una sconcertante gamma di piattaforme diverse, la maggior parte delle quali sono prodotte ad hoc per un progetto specifico, per lo più elaborate da una squadra di sviluppatori specializzati e che quindi risultano difficilmente generalizzabili. Ciò significa che queste edizioni sono risorse molto costose, producibili solo grazie a finanziamenti generosi. Inoltre, le edizioni digitali devono essere necessariamente e ogni volta innovative e diverse, altrimenti potrebbero non ottenere quei finanziamenti che sono necessari alla loro stessa esistenza (Causer, Tonra e Wallace 2012), un requisito che impedisce ulteriormente lo sviluppo di modelli e strumenti stabili e riusabili.

Questi fatti hanno diverse conseguenze: in primo luogo, la loro varietà produce un'oggettiva difficoltà di valutazione da parte della comunità scientifica, con il risultato che le edizioni digitali mancano spesso di riconoscimento come espressioni autorevoli della ricerca ecdotica; in secondo luogo, la creazione di edizioni digitali è possibile solo per quegli studiosi che possono assicurarsi grandi quantità di finanziamenti, escludendo così un gran numero di potenziali ricercatori, soprattutto quelli all'inizio della carriera; in terzo luogo, l'alto livello di variazione di queste risorse, da un lato è causato dalla mancanza di strumenti prontamente disponibili, ma dall'altro ha impedito lo sviluppo di tali strumenti, un fatto che perpetua quindi il problema; infine, la specializzazione di tali edizioni rende la loro conservazione a lungo termine particolarmente complessa e costosa, con il risultato che le edizioni digitali sono percepite come instabili e non degne di investimento e di considerazione accademica.

Questi inconvenienti possono aiutare a spiegare perché così tante edizioni scientifiche sono ancora pubblicate solo su carta, con la conseguenza che i filologi, che sono stati tra i primi e più fini adottatori di metodi digitali, sono ancora oggi combattuti tra il desiderio di sfruttare le opportunità offerte dal supporto digitale e la sicurezza offerta da una pubblicazione cartacea. Quest'ultima, tuttavia, non è esente da problemi, come, per esempio, (1) la comprovata limitazione della pagina rettangolare e la finitezza di ciò che può essere incluso all'interno delle copertine, entrambi fattori non triviali per rappresentare adeguatamente la complessità dei manoscritti d'autore, dei testi modernisti o di grandi tradizioni testuali con centinaia di testimoni; (2) la scarsa circolazione delle edizioni scientifiche al di là del cir- 
colo ristretto che le produce, una scarsità derivante anche dal costo molto alto di tali volumi, spesso accessibili solo dalle biblioteche; (3) la posizione di forza delle case editrici che spesso determinano cosa deve essere pubblicato e come, il che porta a escludere testi di nicchia o percepiti come poco vendibili; (4) la generale mancanza di interesse della comunità scientifica storico-letteraria per la critica testuale.

La filologia digitale è stata vista come un modo per superare questi problemi (Robinson 2003 e 2005), ma mentre alcuni di essi sono stati effettivamente affrontati adeguatamente, altri restano da risolvere. Il problema dei costi sembra il più pressante e quello che ha tenuto lontani molti studiosi, data la mancanza endemica di finanziamenti per le discipline umanistiche (Robinson 2016). Il contrasto tra il modello finanziario di un'edizione basata sulla stampa e uno digitale è alquanto eloquente: $\mathrm{i}$ costi di produzione delle edizioni a stampa sono relativamente bassi, $\mathrm{o}$, più precisamente, i loro modelli finanziari e di lavoro sono integrati nell'infrastruttura di finanziamento della maggior parte delle istituzioni accademiche. Le edizioni a stampa sono spesso prodotte durante il tempo di ricerca "normale" degli studiosi e come tali sono preparate in tempi lunghi: non è insolito che un'edizione richieda dieci o più anni per essere pubblicata; i finanziamenti sono normalmente richiesti per andare in biblioteca, ma ancora una volta, poiché il calendario dell'edizione è abbastanza diffuso, in molti casi questi costi possono essere coperti da indennità di ricerca regolari. In effetti, per gli studiosi è di solito molto più facile accedere a piccole somme di denaro ogni anno, piuttosto che a una grande quantità tutta in una volta. Infine, il sostegno per coprire i costi di pubblicazione può spesso essere ottenuto grazie a specifici fondi universitari o facendo ricorso a fondazioni e/o finanziatori privati. Al contrario, le edizioni digitali hanno normalmente un modello finanziario e lavorativo simile al progetto (Burdick et al. 2012, 130): poiché lo sviluppo tecnico richiede in genere l'assunzione di una forza lavoro specializzata, il lavoro non può essere distribuito come per le edizioni stampate e deve essere concentrato in un tempo relativamente breve (il tempo della disponibilità dei fondi). Questo fatto richiede ai filologi di dedicare una notevole quantità di tempo in modo mirato, e questo a sua volta richiede spesso ulteriori finanziamenti specifici per poter acquistare tale tempo. Mentre questa nuova cronologia potrebbe essere vista per molti aspetti come un miglioramento (con le edizioni che diventano disponibili in modo tempestivo), il lavoro editoriale, tuttavia, non può essere facilmente compresso in questo modo: l'attenzione ai dettagli richiesta per operazioni come trascrizione, collazione e edizione è raggiunta meglio se eseguita in piccole dosi. Inoltre, il brevissimo lasso di 
tempo consentito dalla maggior parte dei sistemi di finanziamento (comunemente da due a tre anni) non consente scoperte di nuovi materiali e/o di gestire le complicazioni che si incontrano spesso mentre si maneggiano manoscritti e altri tipi di fonti primarie; il risultato è che alla fine del finanziamento molte edizioni non sono ancora pronte per il pubblico o non soddisfano gli standard esigenti dei filologi; tuttavia, pubblicate devono essere, poiché i fondi finiscono e ai finanziatori deve essere mostrato che qualcosa è stato fatto con il loro denaro. Il risultato è che molte edizioni sono pubblicate sul web con una dichiarazione di non responsabilità di "Beta" o "Lavori in corso", ad esempio il progetto LangScape (http://www.langscape. org.uk/index.html), sul quale si può vedere Stokes e Pierazzo 2009, e il Vercelli Book (http://vbd.humnet.unipi.it/beta2/). In un articolo precedente (Pierazzo e Leclerc 2015) questo tipo di edizioni è stato definito come edizioni haute couture, con una metafora che viene qui riproposta. ${ }^{1}$

\section{Edizioni baute couture e edizioni prêt-à-porter}

L'industria della moda distingue chiaramente tra due linee di prodotti: l'haute couture, l'alta moda, e il prêt-à-porter. La prima è caratterizzata dal fatto che ogni pezzo è unico e viene spesso creato per una sola persona da indossare per un'occasione speciale, sul tappeto rosso. L'alta moda può e di solito deve essere innovativa e creativa e ha più a che fare con l'arte e l'innovazione che con il produrre articoli indossabili; inoltre l'alta moda produce oggetti di lusso molto costosi e fuori dalla portata della maggior parte di noi. Mentre l'alta moda è presente nei programmi televisivi e nelle riviste patinate, è il prêt-à-porter che, come suggerisce il nome ("pronto da indossare"), le persone possono effettivamente acquistare nei negozi e indossare nella normale vita di tutti i giorni. L'abbigliamento del prêt-àporter è disponibile in diverse taglie e colori e viene normalmente indossato dai suoi possessori più di una volta. Questi articoli possono essere ispirati dall'alta moda, ma la semplificano, rendendola accessibile e portabile.

Se adattiamo questa metafora alle edizioni digitali, notiamo che al momento le edizioni che produciamo sono più simili all'alta moda che al prêt-à-porter: le edizioni digitali sono tipicamente uniche, ognuna è fornita con un set di dedicato di strumenti, è innovativa, creativa, costosa e specifica per il testo per la quale è stata creata e non è normalmente disponibile,

1. La stessa metafora è stata impiegata, indipendentemente, per definire diverse collane di edizioni a stampa, da Elisabetta Risari (2014). 
o difficilmente adattabile, per essere riutilizzata da terzi. Fra tutte queste caratteristiche, il costo è certamente la più problematica: per essere prodotte tali edizioni richiedono personale specializzato (sviluppatori, grafici, web designers ... .), richiedono spazi digitali e server dedicati per mantenerle in vita, richiedono trascrizioni, codifiche, immagini digitali, ecc., ma soprattutto richiedono soldi. La quantità di risorse necessarie le pone molto fuori dalla portata di chi tali fondi non ha e non può avere, come, per esempio, i dottorandi e i ricercatori all'inizio della loro carriera. Esistono certamente all'interno delle varie università dei centri specializzati nelle digital humanites, ma anche questi normalmente lavorano a progetto, vale a dire che spesso bisogna ottenere dei fondi per poter accedere ai servizi, per cui anche per università dove tali centri esistono, la possibilità di creare delle edizioni digitali non è spesso estesa ai giovani ricercatori.

Il fatto che quindi l'accesso agli strumenti della ricerca sia limitato da fattori economici (che spesso si traducono anche in fattori di anzianità) rende la creazione di risorse digitali sofisticate, belle e innovative, non-etica, soprattutto perché esistono poche alternative a questo modello di creazione. Il problema è morale, ma ha ramificazioni profonde e riguarda il futuro stesso della filologia digitale, perché se non possiamo avere dei dottorandi che fanno delle edizioni digitali per mancanza di infrastrutture, se non possiamo dare delle opportunità ai giovani, che sono anche quelli e quelle che hanno una maggior predisposizione per lanciarsi in qualche cosa di nuovo e avventuroso, allora non possiamo formare i filologi del domani e corriamo il rischio di non avere un vero progresso in un settore che ha invece enormi potenzialità scientifiche, ma per il quale abbiamo bisogno di una massa critica di praticanti per poter veramente progredire.

\section{Edizioni seriali e edizioni specializzate}

La sfida è dunque immaginare come potrebbe essere fatta un'edizione prêtà-porter, e quali siano le condizioni scientifiche, tecnologiche e sociali che potrebbero renderla attuabile. Prima di tutto, però, è opportuno pensare a un'appropriata nomenclatura per tali edizioni al fine di non abusare della metafora. Se le caratteristiche della moda prêt-à-porter che abbiamo ritenuto rilevanti nel nostro caso sono il fatto di esistere in un numero limitato di formati, ciascuno di essi modificabili leggermente in un modo controllato, e il fatto di essere poco costosa e accessibile, potremmo forse definire delle edizioni che seguono questo tipo di paradigma come edizioni seriali, mentre potremmo definire le edizioni che seguono il paradigma dell'alta 
moda (uniche, personalizzate, costose) come edizioni specializzate. È importante qui rilevare come tali etichette siano semanticamente neutre, vale a dire che non hanno a priori alcun valore positivo o negativo per sé. In secondo luogo, è anche importante rilevare come immaginare le edizioni digitali del futuro, significa anche immaginare i loro editori, o, meglio, le competenze che questi devono acquisire per poter creare tali edizioni, nonché i loro lettori. Si tratta cioè di una complessa opera di modellizzazione, di cui nelle pagine che seguono si darà solo una sommaria rendicontazione, per evidenti limiti di spazio.

Ma che cosa sono, in pratica, le edizioni seriali e le edizioni specializzate? Il mondo dell'editoria a stampa ha elaborato diversi modelli di edizione, dalle più sofisticate (per esempio i Meridiani Mondadori), alle più generaliste (per esempio gli Oscar Mondadori: Risari 2014); il mondo dell'edito digitale, invece, non è ancora giunto a una chiara definizione dei due approcci. Secondo chi scrive, l'edizione digitale seriale è un'edizione che un ricercatore può creare in modo semplice, senza particolari finanziamenti dedicati, ma che sfrutta i progressi ottenuti dalla ricerca sviluppata per le edizioni specializzate. Per raggiungere questi risultati, una tale edizione non può essere concepita in isolamento, ma deve essere immaginata al plurale, in modo, appunto, seriale. Immagino quindi l'edizione seriale all'interno di una piattaforma editoriale dove ci siano un numero limitato di configurazioni possibili, già pronte all'uso (per esempio: immagine delle fonti sì o no, apparato in una colonna a lato o come pop-up; commento linguistico sì o no, messa in evidenza di nomi di persona e luoghi sì o no, ecc.). La immagino anche situata in un "luogo" (virtuale) predefinito, e che quindi non ci sia bisogno una volta creata, di andare a cercare un server o di occuparsi della sua manutenzione nel tempo. La immagino, insomma, che funzioni da un punto di vista finanziario come un'edizione a stampa.

Quali sono i presupposti per la messa in opera di un tale sistema? Primo, abbiamo bisogno di strumenti informatici che siano capaci di offrire una pubblicazione gradevole e semi-standardizzata del testo, basata su modelli editoriali riconosciuti e riconoscibili (per esempio: edizione documentaria e edizione critica); secondo, abbiamo bisogno di "luoghi" dove questi strumenti e le edizioni generate grazie a questi strumenti possano vivere a lungo. Per quanto riguarda il primo punto, esistono già degli strumenti che aiutano a creare edizioni senza grandi investimenti (uno di questi, EVT, è presentato in questo stesso volume da Roberto Rosselli Del Turco: p. 91), ma questi strumenti sono da un lato troppo pochi per soddisfare tutti i bisogni di ricerca, e dall'altro richiedono comunque una certa dimestichezza con il digitale che non tutti gli editori hanno. Anche solo configurare uno 
strumento come EVT, che è comunque fra i più facili da usare, per aggiungere per esempio qualche funzione che non sia già presente di default, richiede un livello di confidenza con il codice che non è da tutti; è questo che si intende dicendo che non basta pensare a come sarà l'edizione del futuro, ma che bisogna anche pensare a come sarà l'editore del futuro, cioè quali saranno le competenze che dovrà acquisire per usare tali strumenti/ piattaforme: basterà conoscere XML-TEI? O servirà anche l'XSLT e HTML magari corredato da CSS? E poi? JSON? Python? MySQL?2 Per creare uno strumento editoriale bisogna anche proiettare le competenze del suo utilizzatore, e quindi proiettare la pedagogia della filologia del futuro. Di fatto, la maggior parte di queste competenze sono oggi insegnate in diversi corsi di formazione e master specialistici, ma una vera riflessione pedagogica ancora manca e l'inclusione di questa o quella tecnologia segue, in genere, l'intuizione o la preferenza degli organizzatori. A questo si aggiunga il fatto che gli strumenti hanno un forte valore modellizzate, come ci ricorda una frase attribuibile a Marshall McLuhan che recita "we shape our tools, and thereafter our tool shape us"; 3 basti pensare infatti al ruolo preponderante che uno strumento come Microsoft Word ha avuto e sta avendo nel cambiare il nostro modo di scrivere e di concepire la testualità: una volta che lo strumento esiste, sono le sue affordances (vale a dire ciò che esso ci consente di fare e il modo in cui interagiamo con esso) che cambiano il nostro modo di pensare e di agire (Kirschenbaum 2015). Creare degli strumenti editoriali ha quindi delle implicazioni profonde (etiche, epistemologiche, euristiche), vale a dire che quello che gli strumenti ci consentiranno di fare diventerà ciò che faremo, diventerà il confine all'interno del quale concepiremo la nostra attività scientifica. È questo ciò che è accaduto per il libro a stampa: i vincoli della pagina rettangolare, della forma del codex, della

2. Gli acronimi meno universalmente noti si riferiscono, rispettivamente, all'eXtensible Stylesheet Language Transformations (XSLT), al Cascading Style Sheet (CSS), al JavaScript Object Notation (https://www.json.org/) e al sistema di gestione di database relazionali MySQL (https://it.wikipedia.org/wiki/MySQL). Il Python è infine un linguaggio di programmazione di livello medio-alto (https:// www.python.org/).

3. La citazione, generalmente attribuita a McLuhan, in realtà non compare in nessuno dei suoi scritti: appare tuttavia in un articolo su McLuhan scritto da John M. Culkin (1967); l'attribuzione postuma della citazione è incoraggiata anche dagli eredi di McLuhan che nel loro sito ufficiale dichiarano che l'idea è "entirely consistent with McLuhan's thinking on technology in general” (https:// mcluhangalaxy.wordpress.com/2013/04/01/we-shape-our-tools-and-thereafterour-tools-shape-us/). 
collana editoriale, del font tipografico, del costo di riproduzione delle fotografie, ecc., hanno contribuito in modo sostanziale alla definizione dell'edizione scientifica come noi la conosciamo, oltre che al modo di lavorare del filologo e, più importante, alla definizione degli obiettivi scientifici dell'edizione stessa. È quindi cruciale riuscire a creare strumenti che siano basati su analisi scientifiche, basate a loro volta su un consenso costruito attorno a tali analisi. La costruzione di tale consenso non è però un compito facile, come ha notato Tara Andrews (2013): secondo la studiosa i filologi sembrerebbero essere più interessati in ciò che li divide rispetto a ciò che li unisce. Tale valutazione, però, è smentita almeno in parte dall'esistenza stessa dello standard TEI: da più di trent'anni, infatti, tale standard è costruito pezzo per pezzo da un gruppo internazionale di studiosi, per lo più filologi, costantemente rinnovato, che ha prodotto collaborativamente uno dei più raffinati e solidi sistemi di rappresentazione testuale disponibile per il filologo oggi (Pierazzo 2013 e 2016). Le difficoltà certamente ci sono, ma l'esperienza TEI mostra come tale consenso sia in effetti alla portata della comunità scientifica, se opportunamente ricercato.

Per quanto riguarda il secondo aspetto, e cioè il luogo dove predisporre le edizioni seriali, è evidente come l'onere di trovare uno spazio web, acquistare un dominio adeguato, e avere la forza, il tempo e la pazienza di prendersi cura dell'edizione nel corso degli anni non è certamente uno sforzo irrisorio; in un recente intervento Brett Hirsch (2018) ha paragonato l'edizione digitale a un cucciolo che ha bisogno di cure e attenzioni costanti. Lunico modo per ovviare a questi problemi è quello di predisporre delle piattaforme, capaci di ospitare le edizioni e di curarle nel tempo: l'edizione seriale infatti non ha bisogno solamente di spazi di produzione (per i quali abbiamo già qualche strumento utile, come abbiamo visto), ma anche di spazi di pubblicazione sostenibili. La domanda qui è chi dovrebbe produrre tali piattaforme e dove tali spazi potrebbero essere predisposti.

Il candidato più ovvio e quello che più probabilmente potrebbe dare sostenibilità nel tempo a tutta l'impresa, è la biblioteca (vAN Zundert е Воот 2012). Le biblioteche sono istituzioni che si occupano per vocazione della standardizzazione e della conservazione del sapere e quindi sono nella posizione migliore per offrire tali servizi. Esempi come i siti della Biblioteca dell'Università di Cambridge e della Biblioteca dell'Università dell'Indiana a Bloomington (rispettivamente ai link: https://cudl. lib.cam.ac.uk/ e https://libraries.indiana.edu/digital-collections-services) dimostrano questo principio, poiché queste biblioteche si comportano non solo come officine editoriali, supportando e assistendo i ricercatori nello sviluppo di edizioni digitali, ma anche come depositi per quelle edizioni 
digitali che hanno contribuito a modellare e produrre. In Francia il progetto Fonte Gaia, basato presso la biblioteca dell'Università di Grenoble, cerca di seguire questo modello, creando risorse digitali per gli studi italianistici (Pierazzo, Mouraby e Fonio 2016). Ma ci sono altre ragioni per cui le biblioteche e gli archivi potrebbero giocare un ruolo sempre più rilevante nell'ecosistema delle edizioni scientifiche digitali, vale a dire l'uso crescente del protocollo di distribuzione dei facsimili digitali IIIF, acronimo che sta per International Image Interoperability Framework. Tale protocollo consente di usare e riusare all'interno della propria edizione immagini digitali conservate in un qualunque archivio digitale che usa IIIF (tipicamente il sito di una biblioteca); per esempio, se uno studioso ha bisogno di inserire nella propria edizione immagini già disponibili nei siti della Biblioteca Vaticana e della Bibiothèque Nationale de France, basterebbe "richiamare" tali immagini usando il protocollo IIIF, senza doverle scaricare dai rispettivi siti (domandando licenze, pagando i diritti) per poi caricarle nel proprio server. Grazie a questo sistema la biblioteca rimane responsabile per la digitalizzazione e il mantenimento delle proprie risorse, mentre il filologo è libero di occuparsi solamente dell'edizione del testo. Questa evoluzione è relativamente recente (la prima versione del protocollo risale al 2012) ma, come si può capire, potrebbe davvero fornire la chiave di svolta per la creazione di infrastrutture per le edizioni digitali seriali, visto che consente di ridurre notevolmente $\mathrm{i}$ costi di acquisizione e gestione relativamente alle immagini digitali, specialmente ora che le biblioteche stanno digitalizzando il loro patrimonio a ritmo crescente. Secondo Joris van Zundert, però, le potenzialità offerte da IIIF (e dai suoi visualizzatori) non sono ancora completamente sfruttatate dalla comunità scientifica internazionale per un "intellectually hedonistic ideal of publishing the definitive edition", un edonistico ideale intellettuale di pubblicare l'edizione definitiva (2018). La provocazione di van Zundert si lega in parte alla frustrazione provata da Andrews (2013) circa la difficoltà di convincere i filologi a mettersi d'accordo e cambiare completamente il loro modo di lavorare e di pensare. Queste critiche, nemmeno troppo velate, nei confronti dei filologi meriterebbero un'ampia discussione che va oltre lo scopo della presente pubblicazione, ma qualche precisazione merita di essere comunque fatta. Piuttosto che lamentarsi dell'incapacità degli studiosi del settore umanistico di adeguarsi a metodi di ricerca loro estranei, sarebbe forse più produttivo cercare di capire da dove vengono le resistenze e mostrare un maggiore rispetto per metodi e modi di lavoro che spesso hanno tradizioni secolari. Per mettere in pratica quella che ho chiamato l'edizione digitale seriale bisognerà che gli editori scientifici facciano uno sforzo per comprendere un nuovo metodo 
di lavoro, ma per far sì che questo sforzo venga effettuato, bisognerà che esso sia volontariamente e liberamente intrapreso; come ci ricordano Beer (2006) e Ford (2008), la conoscenza e l'interdisciplinarietà sono costrutti sociali, che si sviluppano nel dialogo e nel rispetto reciproco. L'uso di un sistema di edizione distribuita come quello incoraggiato dal protocollo IIIF, se da un lato rende praticabile l'idea di produrre edizioni seriali, dall'altro rappresenta sicuramente un cambiamento nel modo di pensare e concepire il lavoro editoriale che da un insieme chiuso e autosufficiente diventa distribuito e interconnesso. Tale cambiamento non è da sottovalutare sia in termini euristici che pratici, e come tale va pensato collettivamente, nel rispetto delle reciproche tradizioni scientifiche.

Un approccio interessante al problema della creazione di un'infrastruttura per le edizioni seriali è quello offerto da TextGrid, un'infrastruttura finanziata per dieci anni dal governo federale tedesco e ora gestita dalla rete europea DARIAH (Hedges et al. 2013). TextGrid offre alcuni strumenti per l'edizione e per la pubblicazione, ma finora l'infrastruttura è stata utilizzata più per produrre una biblioteca digitale generalista che per produrre singole edizioni scientifiche e gli strumenti di analisi che offre sono molto limitati; tuttavia la sua persistenza e la sua solidità istituzionale rendono TextGrid un buon punto di partenza per quella che chiamiamo un'infrastruttura di edizioni seriali. Vale la pena ricordare qui anche il caso della rivista scientifica digitale Scholarly Editing, che fornisce l'infrastruttura editoriale per la pubblicazione di diverse edizioni all'anno all'interno della serie regolare delle sue pubblicazioni; la rivista ha sede presso l'Università del Nebraska a Lincoln ed è ospitata da uno di questi centri specializzati di DH sopra menzionati (GAILey e Jewell 2012).

Tutti questi esempi condividono una caratteristica molto importante: derivano da istituzioni accademiche e in quanto tali sono in grado di fornire un marchio di rispettabilità alle loro edizioni, un fattore non trascurabile quando si tratta di accettabilità accademica e aspettative di carriera. Oltre a questi approcci istituzionali (biblioteche, riviste e TextGrid), si deve infine menzionare lo sforzo di studiosi come Peter Robinson, uno dei primi ad aver prodotto un software riutilizzabile per le edizioni digitali, vale a dire Anastasia (Robinson 2002 e 2005); in seguito questa esperienza è stata trasformata in una casa editrice chiamata SDE Publisher. Un'altra casa editrice fortemente impegnata nel supportare edizioni digitali scientifiche è la Presses Universitaires de Caen, che ha sviluppato un sistema di organizzazione del lavoro in grado di passare da un file Microsoft Word a una vera e propria edizione scientifica sia online che stampata basata su TEI (BUARD 2015). Sfortunatamente questi due esempi di case editrici sono eccezioni: 
gli editori in generale hanno dimostrato scarso interesse per le edizioni digitali, a meno che queste non siano solo versioni digitalizzate di quelle stampate, cioè delle versioni PDF (si vedano, per esempio le Oxford Scholarly Editions Online e i Cambridge Digital Products). Questo è un fatto particolarmente deplorevole perché le case editrici sono state tradizionalmente responsabili della garanzia della qualità e della diffusione dei prodotti della ricerca filologica. Ė anche vero che il modello editoriale offerto dal digitale e le aspettative del pubblico per l'accesso gratuito a tutte le risorse non sono condizioni che garantiscano un modello commercialmente valido. È possibile però che la creazione di modelli e strutture per le cosiddette edizioni seriali e l'economia dei costi che ciò comporta possa indurre le case editrici a impegnarsi maggiormente nel settore dell'edizione scientifica digitale.

\section{Conclusioni}

Per concludere, alcune parole sulle edizioni specializzate. Come nell'industria della moda, nella filologia digitale abbiamo bisogno sia di haute couture che di prêt-à-porter, di edizioni specializzate e di edizioni seriali, dove le prime hanno il ruolo di sperimentare, di essere innovative, di assumersi il rischio di fallire, mentre le seconde assumono il ruolo di consolidare, di proporre soluzioni scientifiche avanzate per un pubblico più ampio di studiosi, consentendo in particolare a chi è all'inizio della carriera di impegnarsi nella filologia digitale in modo sicuro (sicuro per le loro prospettive di carriera future). È chiaro che l'esistenza dei due approcci alle edizioni digitali non può che essere reciprocamente vantaggiosa, oltre a fornire un mezzo sostenibile di sviluppo per la disciplina. Le sfide che ci attendono sono più accademiche che tecniche, poiché nella pratica esistono già soluzioni tecniche e infrastrutture; ciò che manca è un cambiamento culturale capace di rendere solide e credibili queste iniziative.

Université Grenoble Alpes

\section{Works Cited}

Andrews, Tara. 2013. "The third way. Philology and critical edition in the digital age". Variants 10: 61-76.

BEER, Gillian. 2006. The challanges of interdisciplinarity. Lecture at the Institute of Advanced Studies, University of Durham. https://www.dur.ac.uk/ias/news/annual_ research_dinner/ 
Buard, Pierre-Yves. 2015. Modélisation des sources anciennes et édition numérique. PhD diss., Université de Caen.

Burdick, Anna et al. 2012. Digital_Humanities. Cambridge, MA: The MIT Press.

Cambridge Digital Products. 2019. Cambridge University Press. https://www.cambridge. org/ie/digital-products/

Causer, Tim, Justin Tonra and Valeria Wallace. 2012. "Transcription maximized; expense minimized? Crowdsourcing and editing the collected works of Jeremy Bentham". Literary and Linguistic Computing 27: 19-137.

Culkin, John M. [1967] 2018. “A Schoolman's Guide to Marsahll McLuhan”. Saturday Review 18 (March): 51-53, 71-72.

Ford, Michael. 2008. "Disciplinary authority and accountability in scientific practice and learning". Science Education, 92.3: 404-23.

Gailey, Amanda and Andrew Jewell. 2012. "Editors' introduction to the first issue of scholarly editing: The annual of the Association for Documentary Editing". Scholarly Editing 33: http://scholarlyediting.org/2012/essays/essay.v33intro.html.

Hedges, Mark et al. 2013. "TextGrid, TEXTvre, and DARIAH: Sustainability of Infrastructures for Textual Scholarship". Journal of the Text Encoding Initiative, 5. https://journals.openedition.org/jtei/774.

Hirsch, Brett G. 2018. "Between Puppies and Paper, or When Not To Do a Digital Edition". Oral communication at the symposium "What is Editing in the digital age?", Newcastle University, 12 June: https://research.ncl.ac.uk/atnu/news/whatiseditinginthedigitalage.html.

IIIF. International Image Interoperability Framework. https://iiif.io/

Kirschenbaum, Matthew. 2015. Track Changes: A literary history of word processing. Cambridge, MA: Harvard University Press.

Oxford Scholarly Editions Online. Oxford University Press. http://www.oxfordscholarlyeditions.com/

Pierazzo, Elena. 2013. "Research without borders: the Text Encoding Initiative and international cooperation in digital humanities". Renaissance studies in honor of Joseph Connors, edited by Israëls Machtelt and Louis A. Waldman: 557-62. Florence: Leo S. Olschki - Villa I Tatti, The Harvard Center for Italian Renaissance Studies.

Pierazzo, Elena. 2016. "Textual scholarship and text encoding”. A New Companion to Digital Humanities, edited by Susan Schreibman, Ray Siemens, and John Unsworth: 307-21. Oxford: Wiley Blackwell.

Pierazzo, Elena and Elise Leclerc. 2015. "Ledizione scientifica al tempo dell'editoria digitale". Ecdotica 12: 180-94.

Pierazzo, Elena, Claire Mouraby, and Filippo Fonio. 2016. "A new approach to libraries in Digital Humanities: the case of Fonte Gaia". Digital Humanities 2016, Kraków: Jagiellonian University and Pedagogical University, Conference Abstracts: 862-3.

Risari, Elisabetta. 2014. "Haute couture e prêt-à-porter. Cassola e altri scrittori italiani del secondo Novecento tra Meridiani e Oscar". Studi e Testi 33: 103-9. 
Robinson, Peter M. W. 2002, Anastasia: Analytical System Tool and SGML/XML Integration Applications. Software. http://sd-editions.com/anastasia/index.html.

. 2003. "Where we are with electronic scholarly editions, and where we want to be". Computerphilologie 5: http://computerphilologie.tu-darmstadt.de/jg03/robinson. html.

2005. "Current issues in making digital editions of medieval texts — or, do electronic scholarly editions have a future?" Digital Medievalist 1: http://doi. org/10.16995/dm.8.

_ 2016. "Project-based Digital Humanities and social, digital, and scholarly editions". Digital Scholarship in the Humanities 31.4: 875-89.

Stokes, Peter A. and Elena Pierazzo. 2009. "Encoding the language of landscape: XML and databases at the service of Anglo-Saxon lexicography". Perspectives on lexicography in Italy and Europe, edited by Silvia Bruti, Roberta Cella, and Marina Foschi Albert, 203-38. Newcastle: Cambridge Scholars Publishing.

TEI Consortium, eds. 2018. Guidelines for Electronic Text Encoding and Interchange. V. 3.3.0. http://www.tei-c.org/P5/.

van Zundert, Joris. 2018. "On not writing a review about Mirador: Mirador, IIIF, and the epistemological gains of distributed digital scholarly resources". Digital Medievalist 11/1: http://doi.org/10.16995/dm.78 .

van Zundert, Joris and Peter Boот. 2012. "The digital edition 2.0 and the digital library: Services, not resources”. Bibliothek und Wissenschaft 44: 141-52. 